\title{
Impactos socioambientais da construção da usina hidrelétrica no município de Tucuruí e seus reflexos sobre a malária
}

Socio-environmental impacts of construction of the hydroelectric plant in the municipality of

Tucuruí and its reflections on malaria

\begin{abstract}
Impactos socioambientales de la construcción de la hidroeléctrica en el municipio de Tucuruí y sus reflejos en la malaria
\end{abstract}

Herberth Rick dos Santos Silva ${ }^{1 *}$, Leonardo Gomes de Sousa1, Wendrel Gonçalves Furtado', Benedito do Carmo Gomes Cantão ${ }^{1}$, Jaylen França Cunha ${ }^{1}$, Ailson Almeida Veloso Júnior ${ }^{1}$, Eunice Lara dos Santos Cunha ${ }^{1}$, Artur Chaves Cruz ${ }^{1}$, Anderson Bentes de Lima ${ }^{2}$, Ana Gabriela Sabaa Srur de Andrade ${ }^{3}$.

\section{RESUMO}

Objetivo: O estudo tem como objetivo analisar a influência dos fatores socioambientais decorrentes da construção da Usina Hidrelétrica de Tucuruí e seus impactos na malária, por meio da análise do perfil epidemiológico dos casos de malária no ano de 2012-2018 em Tucuruí. Métodos: Trata-se de um estudo com abordagem quantitativa de caráter epidemiológico observacional do tipo analítico de delineamento ecológico retrospectivo, realizando uma investigação de dados epidemiológicos da malária no município de Tucuruí. A população do estudo fora casos notificados de malária durante os anos de 2012 a agosto de 2018 no âmbito urbano e rural do município de Tucuruí-PA. Resultados: Constatou-se que a atividade da Usina Hidrelétrica de Tucuruí na região, implicou diretamente no aumento agravos de saúde pública na comunidade em questão, como: aumento no número de mosquitos, realocação populacional devido as inundações, aumento na ocorrência de novos casos de malária. Conclusão: Os objetivos do trabalho foram alcançados, sendo possível formar o perfil epidemiológico dos casos notificados de malária em Tucuruí, bem como correlaciona-los à construção da UHE segundo a literatura sobre o assunto, assim, concluindo que há paralelos diretos entre a prevalência de Malária local com a presença da Hidrelétrica local.

Palavras-chave: Epidemiologia, Malária, Meio Ambiente.

\begin{abstract}
Objective: The study aims to analyze the influence of socio-environmental factors resulting from the construction of the Tucuruí Hydroelectric Power Plant and its impact on malaria, through the analysis of the epidemiological profile of Malaria cases from the year 2012-2018 in Tucuruí. Methods: This is a study with a quantitative approach of an observational epidemiological nature of the analytical type of a retrospective ecological design, carrying out an investigation of epidemiological data on malaria in the municipality of Tucuruí. The study population had been reported cases of malaria during the years 2012 to August 2018 in urban and rural areas of the municipality of Tucuruí-PA. Results: It was found that the activity of the Tucuruí Hydroelectric Plant in the region, directly implied in the increase of public health problems in the community in question, such as: increase in the number of mosquitoes, population relocation due to floods, increase in the
\end{abstract}

1 Universidade do Estado do Pará (UEPA).Tucuruí - PA. *E-mail: rick.santos.hr@gmail.com

2 Universidade Federal do Pará (UFPA.) Belém - PA.

${ }^{3}$ Fundação Santa Casa de Misericórdia do Pará. Belém - PA.

SUBMETIDO EM: 5/2020 
occurrence of new cases of malaria. Conclusion: The objectives of the work were achieved, making it possible to form the epidemiological profile of the reported cases of malaria in Tucuruí, as well as to correlate them to the construction of the HPP according to the literature on the subject, thus concluding that there are direct parallels between the prevalence of Local malaria with the presence of the local hydroelectric plant.

Key words: Epidemiology, Malaria, Environment.

\section{RESUMEN}

Objetivo: Realizar una encuesta analítica de un diseño ecológico retrospectivo (2012-2018), utilizando como base los datos contenidos en el Sistema de Vigilancia e Información Epidemiológica de la Malaria (SIVEPMalaria). Métodos: Este es un estudio con un enfoque cuantitativo de naturaleza epidemiológica observacional del tipo analítico de un diseño ecológico retrospectivo, que lleva a cabo una investigación de datos epidemiológicos sobre la malaria en el municipio de Tucuruí. La población del estudio había sido reportada de casos de malaria durante los años 2012 a agosto de 2018 en áreas urbanas y rurales del municipio de Tucuruí-PA. Resultados: se encontró que la actividad de la Central Hidroeléctrica Tucuruí en la región, directamente implicada en el aumento de los problemas de salud pública en la comunidad en cuestión, tales como: aumento en el número de mosquitos, reubicación de la población debido a inundaciones, aumento en la aparición de nuevos casos de malaria. Conclusión: Se lograron los objetivos del trabajo, lo que permitió formar el perfil epidemiológico de los casos reportados de malaria en Tucuruí, así como correlacionarlos con la construcción del HPP según la literatura sobre el tema, concluyendo así que existen paralelos directos entre la prevalencia de Paludismo local con presencia de la planta hidroeléctrica local.

Palabras clave: Epidemiología, Malaria, Ambiente.

\section{INTRODUÇÃO}

A Usina Hidrelétrica de Tucuruí (UHE - TUC) fica localizada na região sudeste do Pará, na distância de $310 \mathrm{~km}$ da capital. A mesma, começou a ser projetada no governo do general Emílio Garrastazu Médici em 1973 e foi inaugurada em 1984, sendo destinada ao abastecimento de recurso elétrico para a expansão de grandes mineradoras do país, tais como: Companhia Vale do Rio Doce, Albrás, Alunorte e Alumar (PINTO LF, 2012). A estruturação de Usinas Hidrelétricas de Energia (UHE) culmina em inúmeras indagações relacionadas ao custo-benefício, consentâneo que tais empreendimentos trazem intensas transformações na vida da comunidade local (FEARNSIDE PM, 2015).

Agostinho AA, et al. (2007) observou, em seu livro, que os impactos socioambientais são perceptíveis e são demonstrados diretamente na saúde coletiva do município supracitado. $O$ autor, também explana que com o represamento de um rio, a hidrologia local é completamente alterada, passando de um estado lótico para uma condição lêntica ou semilêntica, o que significa que as condições químicas e físicas da água serão alteradas, assim como a qualidade e a quantidade de hábitats para a fauna e flora da hidrosfera, principalmente pelas mudanças nas relações tróficas, na base da produção primária e no ciclo de nutrientes.

Pantoja GMT e Andrade RF (2012), em seus estudos, observaram que no espectro das consequências oriundas da energia gerada pelas Hidrelétricas, está o aumento na prevalência de doenças vetoriais, entre os demais agravos à saúde humana. Tal fato, dá-se por conta do desflorestamento em larga escala, formação de reservatórios, modificações socioambientais, formação de mazelas sociais, entre outros. Entretanto, a malária necessita de maior atenção por ser associada diretamente a construção de empreendimentos do setor elétrico especialmente em região tropical.

A malária configura-se como expoente problema de saúde pública no cenário mundial, estando presente em 106 países e territórios, ameaçando aproximadamente 50\% da população global. Calcula-se que ocorram de 300 a 500 milhões de novos casos com percentual de um milhão de mortes por ano, em sua grande maioria crianças menores de cinco anos e gestantes de origem africana, bem como imigrantes oriundos de área não endêmicas (MONTE CRS; MIRANDA AMM; OLIVEIRA MHC, 2012). 
A malária é uma doença infecciosa febril aguda causada por protozoários do gênero Plasmodium que se reproduzem nos eritrócitos (células vermelhas do sangue) do hospedeiro, sendo transmitida pela picada da fêmea do mosquito Anopheles infectado pelo Plasmodium. Seguida a inoculação, o ciclo continua, onde os parasitos seguirão para as células do fígado (hepatócitos) e irão se replicar, gerando centenas de outros patógenos (merozoítos) que romperão os hepatócitos dando início à segunda fase do ciclo denominada de esquizogonia sanguínea. No Brasil, as espécies P. vivax, P. falciparum e P. malarie são as principais expoentes. Se a doença não for tratada adequadamente, pode evoluir para a forma grave, com febre superior a $41^{\circ} \mathrm{C}$, hiperparasitemia, anemia intensa, icterícia, hemorragias e hipotensão arterial, levando a coma e óbito (BRASIL, 2010; REY L, 2010).

Referente ao diagnóstico, Brasil (2010) aponta que a microscopia da gota espessa do sangue é o método mais utilizado. Neste, é possível detectar densidades de até 5-10 parasitos por $\mu l$ de sangue. Contudo, nas condições de campo, a capacidade de detecção torna-se de 100 parasitos $/ \mu$ le sangue.

Focaccia R e Veronesi R (2015) apontam que o objetivo imediato do tratamento da malária é abolir o ciclo de reprodução sanguínea do parasita responsável pelas manifestações clínicas agudas da doença e pelas eventuais complicações. Quando iniciado precocemente e utilizado de maneira correta, é altamente eficaz. Além desse objetivo, o tratamento também possibilita a erradicação das formas latentes no ciclo tecidual evitando as recaídas tardias. Outro importante objetivo do tratamento adequado é a eliminação de gametócitos, permitindo a interrupção da transmissão em áreas endêmicas.

Fearnside PM (2015), expõe que no que tange a correlação de doenças vetoriais, como a malária, à construção de usinas, os impactos socioambientais gerados pela UHE-TUC eram pré-vizualizados, já que o preenchimento do reservatório acarretaria na substancial elevação do número de mosquitos do gênero Mansonia, no entorno do lago, em virtude dos macrófitas aquáticas que fornecem criadouros ideais para estes vetores, em toda região amazônica. O referido autor ressalta, também, que o represamento do rio Tocantins foi um dos fatores determinantes para a alta prevalência de casos notificados de malária no local.

Dentro do espectro da visualização epidemiológica, Kirchhof ALC (2012) afirma, em seu estudo, que a enfermagem ao da epidemiologia, pode desenvolver estudos de prevenção, promoção e avaliação do cuidado, em diversas áreas, como o planejamento de serviços de saúde, a construção de programas e estratégias, e também a investigação e controle de processos de saúde coletiva.

Vicente JA (2016), afirma que as pesquisas em torno da temática supracitada têm proporcionado avanços positivos na área, visto que o uso da epidemiologia pelo enfermeiro torna-se um instrumento fundamental à prestação de assistência à saúde, onde, o profissional poderá propor intervenções educativas com mais autonomia e desenvolver maior senso crítico a respeito dos impactos de forma global. Diante disso, o presente artigo tem como objetivo analisar a influência dos fatores socioambientais decorrente da construção da Usina Hidrelétrica de Tucuruí e seu reflexo na malária, através da análise do perfil epidemiológico dos casos de Malária no recorte temporal de 2012-2018 em Tucuruí.

\section{MÉTODOS}

Trata-se de um estudo com abordagem quantitativa de caráter epidemiológico observacional do tipo analítico de delineamento ecológico retrospectivo, onde foi realizado por meio de uma investigação retrospectiva de dados epidemiológicos da malária no município de Tucuruí, que incluíra uma análise geoestatística paralela à geração de hipóteses etiológicas a respeito das ocorrências dos impactos socioambientais causado pela construção da hidrelétrica de Tucuruí e seus reflexos na malária.

Os dados secundários referem-se aos casos novos de malária notificados no município de Tucuruí-PA e compreendem uma série histórica de quase 6 anos (2012 a 2018), recorte temporal, este escolhido, por serem os anos em que os dados se preservaram íntegros, sem intercorrências de perdas que viessem a atrapalhar a compreensão da análise. O estudo epidemiológico ecológico foi realizado na Fundação Nacional de Saúde - FUNASA e no Sistema de Informação de Vigilância Epidemiológica - SIVEP, localizados no município de Tucuruí. Estes locais foram escolhidos por serem departamentos responsáveis pelos registros de casos de 
malária do gênero falciparum e vivax que ocorreram no período de 2012 a agosto 2018 no município de Tucuruí - Pa. Houvera previamente a solicitação, a partir de ofício institucional, para a autorização da coleta de dados do banco de dados do SIVEP. A população em estudo fora a de casos notificados e confirmados de malária durante os anos de 2012 a 2018 no município de Tucuruí-PA. O estudo tivera como variantes o sexo biológico e a origem do paciente, sendo esta, rural ou urbana. Para a exploração epidemiológica e análise dos casos de malária em Tucuruí nos anos de 2012 a 2018, foram utilizados os programas Microsoft Office Word 2016, Microsoft Office Excel 2016 e BioEstat 5.3.

Os critérios de inclusão, foram: compreensão de dados epidemiológicos íntegros; dados fornecidos pela FUNASA E SIVEP; e obtidos através de notificações individuais de casos confirmados de malária. Os dados de exclusão foram: dados de casos suspeitos ou negativos; dados de outros estudos; e dados de outro município.

O presente trabalho não necessitou de envolvimento do Comitê de Ética em Pesquisa em razão da característica aberta dos dados, impossibilitando qualquer forma de identificação dos indivíduos envolvidos, além de não utilizar material biológico decorrente desses, conforme preconiza a Resolução 466/12, do Conselho Nacional de Saúde (OLIVA HNP, et al., 2018).

\section{RESULTADOS E DISCUSSÃO}

No Brasil, a região amazônica obtém o maior número de casos de malária, devido às condições ambientais e socioculturais que potencializam a transmissão e disseminação da mesma. Por exemplo, em 2010 cerca de 97\% dos casos estavam distribuídos em seis estados da região em questão: Acre, Amapá, Amazonas, Pará, Rondônia e Roraima, sendo o Pará a região que apresentou mais morbidade pela endemia (BRASIL, 2010).

Avaliou-se um total de 3.671 casos de malária no município de Tucuruí-PA entre os anos de 2012 a agosto de 2018. As avaliações tiveram como base os dados do SIVEP - Malária, que serviram como base para traçar o perfil epidemiológico da doença em estudo. $\mathrm{O}$ ano de 2012 fora o maior expoente no que tange ao número de casos notificados, tendo registrado $1563(42,2 \%)$ casos confirmados de malária, seguido pelo ano de 2018, que até o mês de agosto, já houvera $766(20,8 \%)$ casos confirmados. Dentre os anos com menores casos, figura o ano de 2014, tendo 109 (2.9\%) casos confirmados e 2015, com $163(4,4 \%)$ (Gráfico 1).

Gráfico 1 - Frequência anual de casos de Malária no município de Tucuruí entre os anos de 2012 a agosto de 2018.

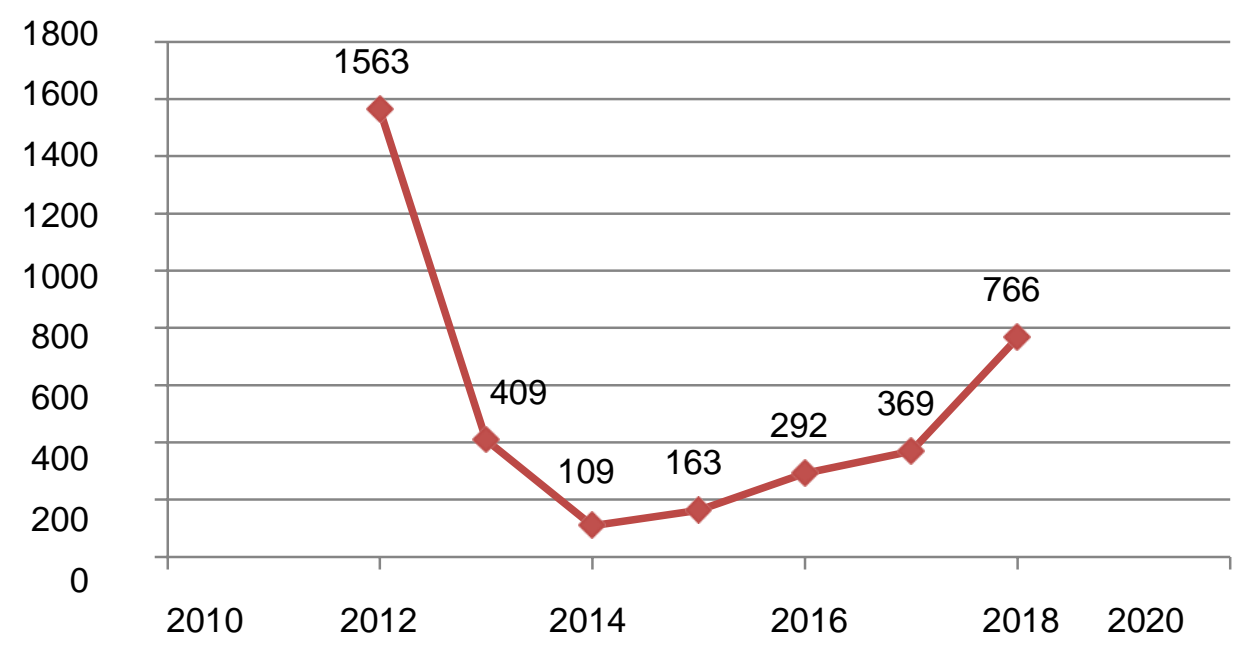

Fonte: Silva HRS, et al., 2020; dados extraídos de SIVEP - Malária.

Segundo Suarez-Mutis MC e Coura JR (2007), a incidência da malária varia de mês para mês e de ano para ano, o que vêm a justificar o alto número de casos no ano de 2012, no estudo. $O$ autor, afirma que se apresentam surtos epidêmicos estacionais que podem chegar a ser de grandes proporções quando as 
condições climáticas são ótimas, com grandes variações que decorrem por causas inaparentes. Têm sido registrados ciclos com períodos de 5 a 8 anos. $O$ grau de imunidade da população de áreas amazônicas é muito variável. Nesses lugares, a intensidade da transmissão não permite que as pessoas desenvolvam um alto grau de imunidade naturalmente adquirida e, portanto, não chega a ser protetora.

Esse quadro foi influenciado pelo fato do estado do Pará ser uma área endêmica para malária e por apresentar características biológicas, ecológicas, geográficas e socioeconômicas que facilitam a transmissão da doença e Tucuruí possuir características homologas a de outros municípios do estado do Pará (PARISE et al., 2009).

Segundo Fearnside PM (2015), a construção da UHE-TUC, influenciara os processos migratórios para o município, favorecendo a proliferação de endemias, já que na Amazônia, a migração e malária são problemas previsíveis, devido à relação entre frequência de malária e o uso da terra, ou seja, surgem as consequências da forma como o homem utiliza o meio para produção de subsistência.

Dessa forma, ocorre a ruptura do equilíbrio ecológico pré-existente perante a forma de uso da terra, a qual apresenta especificidades que influenciam os níveis de proliferação de malária. Também há as interferências nos quesitos socioculturais, nos quais dá-se a formação de mazelas sociais, influenciadas pelo processo migratório, formando assim, condições específicas, como a moradia irregular, para o processo de proliferação de doenças vetoriais.

Como demonstrado pelo estudo de Oliveira AB e Martinelli JM (2009), os dados dos municípios, incluindo Tucuruí, do estado do Pará, tido como o estado de maior incidência de malária, são aqueles que tiveram elevadas taxas de crescimento populacional, com aglomerados espontâneos em áreas de florestas, sem nenhuma infraestrutura urbana, favorável à exposição de contingente populacional aos vetores da doença.

Assim, o número de casos de malária no município de Tucuruí de fato é correlacionado com a construção da UHE-TUC. Como se reconhece por estudos como o de Kibret S, et al. (2015), a modificação ambiental provocada pela construção de usinas hidrelétricas, aumenta a transmissão de patologias como a malária, na qual a mesma doença afeta globalmente cerca de 627.000 vidas a cada ano.

Na seguinte variável analisada, que refere-se a frequência segundo a espécie da malária no período de 2012 a agosto de 2018, verifica-se que a infecção ocasionado pelo P. vivax é exorbitante, com cerca de 3.415 $(92,9 \%)$ do total de casos, em comparação ao P. falciparum que corresponde somente a $242(6,6 \%)$ casos e a mista (falciparum+ vivax) que fora de $13(0,5 \%)$, casos estes, confirmados e notificados, entre 0 ano de 2012 a agosto 2018 no município de Tucuruí-PA (Gráfico 2).

Gráfico 2 - Frequência total dos casos de malária no município de Tucuruí-PA, no período de 2012 a agosto de 2018, quanto às espécies de Plasmodium.

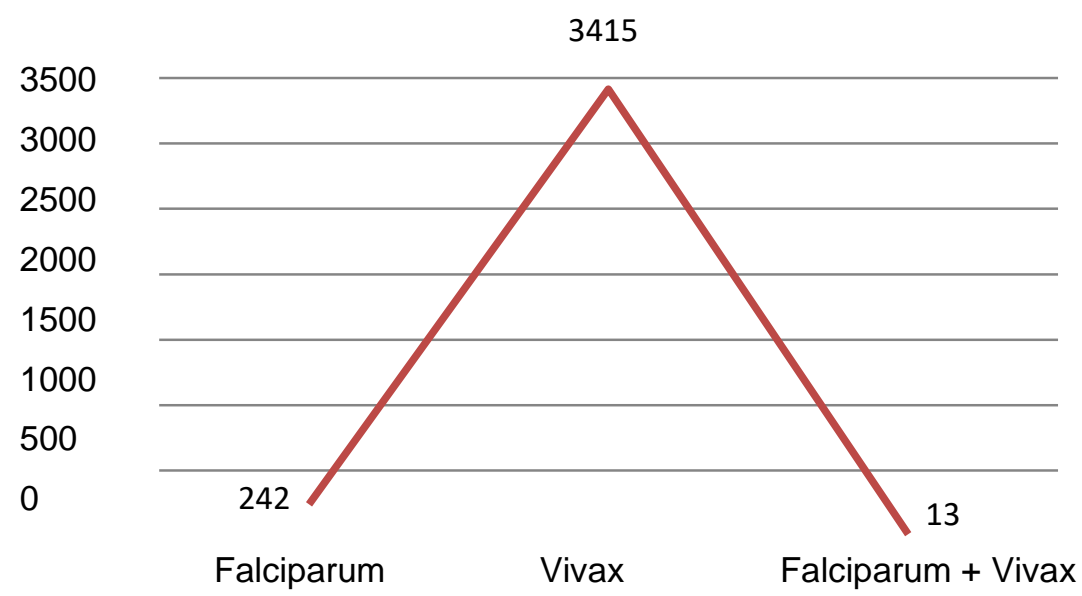

Fonte: Silva HRS, et al., 2020; dados extraídos de SIVEP - Malária. 
Segundo Rodrigues PT (2017) em 2014 o P. vivax foi responsável por 142 mil casos de malária em todo o país. Esses dados podem ser explicados por fatores que estão ligados com a presença de estágios hepáticos dormentes, conhecido como hipnozoítos, assim como pela circulação sanguínea precoce de estágios sexuais denominados de gametócitos. Tornando assim o P. vivax menos sensível em comparação ao P. falciparum em relação ao arsenal de controle disponível (medicamentos).

De acordo com Coura JR, et al. (2006), apesar do P. Vivax ser hoje considerado predominante a nível nacional, na década de 70 a 80 a espécie de maior incidência era o P. falciparum. Entre o ano de 1966 a 1988 o P. falciparum prevaleceu com mais de $50 \%$ dos casos sendo a prevalência atribuída principalmente ao acelerado e desenfreado processo migratório, expansão das atividades garimpeiras, desmatamentos ilegais em alta escala e projetos de assentamentos. O P. vivax começou a predominar a partir de 1988 saindo de $48,6 \%$ para mais de $80 \%$ atualmente.

No que tange a variável da idade, na pesquisa observou-se que a predominância da frequência de casos de malária, encontrou-se na faixa etária entre 20 a 29 e 30 a 39 anos, registrando um total de 1.323 casos na soma das duas variáveis no período de 2012 a 2017. Ao correlacionar às duas faixas etárias de maior frequência, chegou-se a uma estimativa de $50,9 \%$ em relação ao total de caso (Gráfico 3).

Gráfico 3 - Frequência total dos casos de malária no período de 2012 a 2017, no município de Tucuruí - PA, segundo a idade.

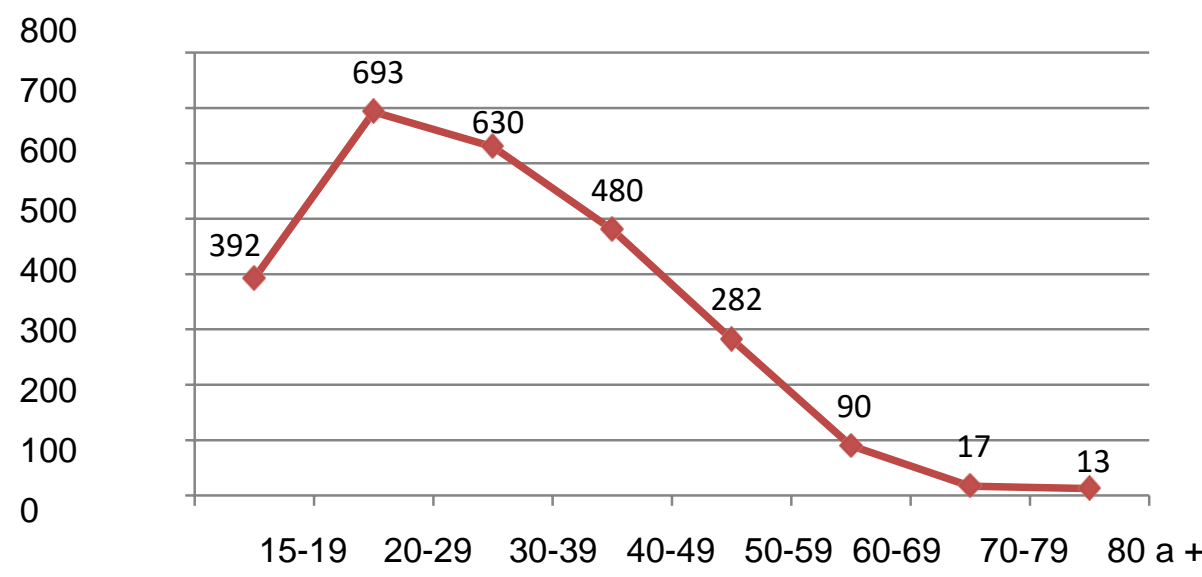

Fonte: Silva HRS, et al., 2020; dados extraídos de SIVEP - Malária.

O pico considerável na faixa etária dos 20 aos 50 anos é reflexo do deslocamento de mão-de-obra, na busca por novas oportunidades de emprego, em áreas onde prevalece atividades voltadas para o setor agrícola, pecuária, caça/pesca garimpo e exploração vegetal (BRASIL, 2008).

Segundo Parasi EV (2009) é inquestionável que esse fato está relacionado com a exposição precoce de indivíduos acima de 14 anos a ambientes propícios a presença de vetores contaminados. Pois, a grande maioria dos jovens necessita entrar na cadeia produtiva, passando assim a responder por um fenômeno social, no qual os mesmos adquirem sua independência financeira e familiar.

O presente estudo está de acordo com outras investigações epidemiológicas locais. O estudo de Filho AS, et al. (2020) realizado em nove cidades do Pará, em um período de 21 anos, assegurou que, em seus resultados, $30 \%$ da amostra positiva para malária, tinha entre 19 - 30 anos, seguida pela faixa etária de 21 40 , que apresentou $22 \%$ do total de casos estudados.

Dando continuidade à apresentação de dados, dos 3.796 casos notificados entre o período de 2012 a 2017, destes $2.635(69,4 \%)$ foram pacientes do sexo masculino e $1.161(30,6 \%)$ do sexo feminino, com idade entre 15 e 80 anos. Pode-se observar que os indivíduos do sexo masculino predominam durante toda série histórica como os mais acometidos pela doença, demonstrando um perfil constante na distribuição ao longo dos anos (Gráfico 4). 
Gráfico 4 - Frequência total dos casos de malária no período de 2012 a 2017, no município de Tucuruí - PA, segundo o gênero.

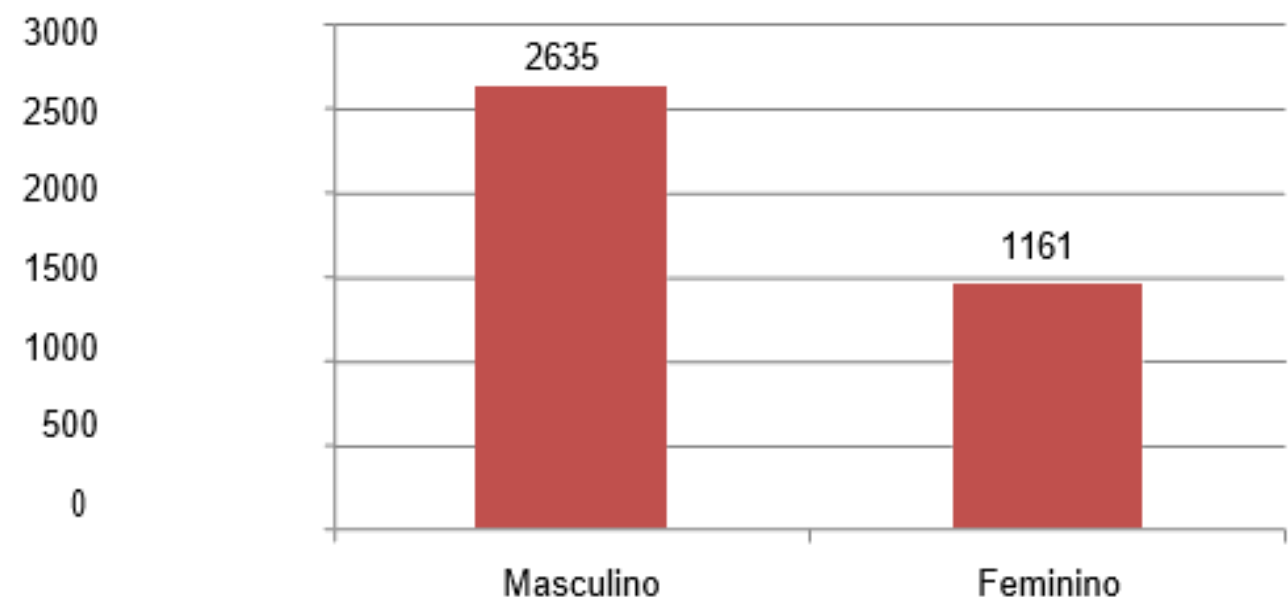

Fonte: Silva HRS, et al., 2020; dados extraídos de SIVEP - Malária.

Segundo Parise EV (2009), em geral, todo indivíduo é susceptível à infecção por malária. Contudo, pessoas de determinado sexo e idade podem ter probabilidades diferenciadas de serem infectadas pela patologia, que pode estar relaciona da com o tipo de ocupação, a mobilidade, e/ou ambiente em que residem.

O referido autor também afirma que os processos que levam o gênero masculino a desenvolver a endemia em maior porcentagem que as mulheres, estão relacionadas com a inserção precoce do mesmo nas atividades produtivas, ou seja, desenvolvimento de trabalhos que necessitam de maior deslocamento para áreas rurais, onde a malária se apresenta de forma endêmica.

Yeshiwondim AK, et al. (2009) ressaltam que o elevado índice de malária no sexo masculino em comparação com as do sexo feminino está vinculado com as diferentes taxas de exposição ao vetor, maior mobilidade, desenvolvimento de atividades relacionadas com trabalho no campo, além da permanência em áreas de maior risco de transmissão (zona rural). No que tange a zona de contaminação, o estudo demonstra que houve uma disparidade expressiva no número de notificações da doença na zona rural comparada a zona urbana, pois de um expoente de 3.671 casos registrados no intervalo de 6 anos, 3.524 (96\%) são da zona rural e penas $146(4 \%)$ da zona urbana (Gráfico 5).

Gráfico 5 - Percentual de casos de malária no período de 2012 a 2017, no município de Tucuruí-PA, por zona.
- Zona rural
- Zona urbana

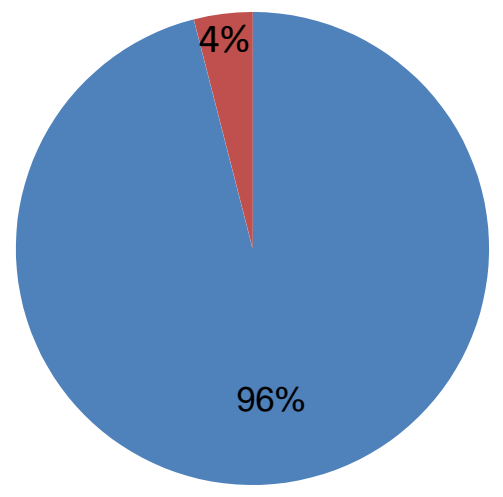

Fonte: Silva HRS, et al., 2020; dados extraídos de SIVEP Malária. 
O estudo demonstra que houve uma disparidade expressiva no número de notificações da doença na zona rural comparada a zona urbana, pois de um universo de 3.671 casos registrados no intervalo de 6 anos, $96 \%$ são da zona rural e penas $4 \%$ da zona urbana. Sarmento RA, et al. (2015) diz que esse fato está relacionado com as características peculiares da zona rural, tais como: desigualdade socioeconômica, insuficiência do sistema de esgoto, abastecimento de água insatisfatório e outros, logo, essa localidade se destaca em comparação a zona urbana, por conta do elevado índice de doenças de veiculação hídrica e vetoriais, devido os indivíduos viverem em condições propícias para inúmeras doenças, entre elas a malária.

Sarmento RA, et al. (2015), também, afirma também que o número elevado de casos na zona rural está ligado com a permanência dos indivíduos no habitat natural do vetor, a floresta, e sua derrubada, como acontece em grandes modificações ambientais, como a implantação de hidrelétricas, implica em alteração de toda a dinâmica do processo, em particular em doenças vetoriais como a malária.

Brasil (2015) reforça que a malária ocorre de maneira mais frequente em ambiente rural, contudo, com o passar dos anos vem ocorrendo um processo denominado de "urbanização da malária", ou seja, a cada ano é quantificado um percentual maior de casos da doença, principalmente em grandes centros urbanos. Em comparação ao estudo de Suarez-Mutis MC e Coura JR (2007), houvera concordância com os dados encontrados no presente estudo. No supracitado, em um período de cinco anos, os maiores índices notificados fora os registrados na área rural. Ressalta-se à necessidade de prover medidas que sigam além da assistência, o que inclui pesquisas e intervenção em saúde, assim como formação e qualificação profissional. É necessário um delineamento das políticas e programas de controle, e no caso da malária, compreender tal patologia é de suma importância para atender às necessidades da população (NASCIMENTO TL, et al., 2019).

Segundo Mourão FR, et al. (2014) os sistemas de vigilância estabelecidos pelas autoridades nacionais e internacionais de saúde pública devem ser capazes de enfrentar qualquer desafio de evento anormal de saúde, conhecidos ou desconhecidos ou ocorridos naturalmente, como a malária. O surgimento de novas ferramentas no campo da epidemiologia (redes de computadores, vigilância sanitária, sistemas de informação, testes de diagnóstico rápido, etc.) melhoraram as práticas de detecção de surtos. Os dados de vigilância e monitoramento em níveis nacionais são cruciais para saber quando as metas são cumpridas e, ainda mais importante, para iniciar correções no meio do percurso se o progresso não for suficiente. A necessidade de acompanhamento global e um plano de avaliação tem sido reconhecida pelos doadores e agências técnicas internacionais.

\section{CONCLUSÃO}

Fora constatado ao longo da pesquisa bibliográfica, por inúmeros autores, que a construção da UHE-TUC implicou em diversos agravos de saúde para a comunidade, tais como: elevação de mosquitos transmissores de doenças vetoriais, deslocamento da população por questões socioeconômicas, aumento na ocorrência de malária, dentre outros fatores que influenciam diretamente na vida da população local. Com relação aos números de casos de malária analisados entre o ano de 2012 a agosto de 2018, constatou-se que em 2012 houvera a maior frequência de casos. Referente a espécie do gênero do patógeno da malária, embora o Plasmodium falciparum tenha manifestado leve progressão na proporção entre as espécies, o Plasmodium vivax manteve-se na predominância durante todo do o histórico epidemiológico. O perfil epidemiológico da malária se apresentou da seguinte forma: atingiu em maior quantidade a zona rural, em indivíduos do sexo masculino, entre a faixa de 20 a 29 anos. Os objetivos do estudo foram alcançados, sendo possível a realização do reconhecimento dos casos notificados no cenário do estudo, bem como da relação dos impactos socioambientais da construção da usina hidrelétrica no município de Tucuruí e seus reflexos sobre a malária.

\section{REFERÊNCIAS}

1. AGOSTINHO AA, et al. Ecologia e manejo de recursos pesqueiros em reservatórios do Brasil. Maringá - PR: Editora da Universidade Estadual de Maringá, 2007; 43.

REAS/EJCH | Vol.12(10) | e3945 | DOI: https://doi.org/10.25248/reas.e3945.2020 Página 8 de 9 
2. BRASIL. Ministério da Saúde, Secretaria de Vigilância em Saúde. Boletim Epidemiológico, 2015; 46- 43.

3. BRASIL. Ministério da Saúde. Secretaria de Vigilância em Saúde. Departamento de Vigilância Epidemiológica. Guia prático de tratamento da malária no Brasil/ Ministério da Saúde, Secretaria de Vigilância em Saúde, Departamento de Vigilância Epidemiológica. - Brasília: Ministério da Saúde, 2010; 10 - 11.

4. BRASIL. Ministério da Saúde. Secretaria de Atenção à Saúde, Departamento de Atenção Básica. Vigilância em Saúde: dengue, esquistossomose, hanseníase, malária, tracoma e tuberculose. rev. Brasília, 2008; 113- 119.

5. COURA JR, et al. A new challenge for malaria control in Brazil: asymptomatic Plasmodium infection-a review. Memórias do Instituto Oswaldo Cruz, 2006; 101(3): 229-237.

6. FEARNSIDE PM. Hidrelétricas na Amazônia: impactos ambientais e sociais na tomada de decisões sobre grandes obras. Editora do INPA, Manaus, 2015; 1(1): 296.

7. FILHO SA, et al. Análise dos aspectos clínicos, epidemiológicos e parasitológicos de indivíduos com malária falciparum: análise dos aspectos clínicos, epidemiológicos e parasitológicos de indivíduos com malária falciparum. Editora Atena, 2020; 1: 29-43.

8. FOCACCIA R e VERONESI R. Tratado de Infectologia - Vol 1. 5a ed. São Paulo: Editora Atheneu, 2015.

9. KIBRET S, et al. Malaria impact of large dams in sub-Saharan Africa: maps, estimates and predictions. Malaria journal, 2015; 14(1): $339-349$.

10. KIRCHHOF ALC. Epidemiologia e a pesquisa na saúde e na enfermagem. Cogitare Enfermagem, 2009 (14):2.

11. MONTE CRS, et al. Endemicidade da Malária nos Municípios de Abrangência da UHE Belo Monte no Período de 2003 a 2011. Congresso Norte e Nordeste de Pesquisa e Inovação. 2012, Anais CONNEPI, 2012; 1.

12. MOURÃO FR, et al. A Vigilância Da Malária Na Amazônia Brasileira. Biota Amazônia. Macapá, 2014; 4(2): $161-168$.

13. NASCIMENTO TL, et al. Prevalence of malaria relapse: systematic review with meta-analysis. Revista latinoamericana de enfermagem, 2019; 27.

14. OLIVA HNP, et al. Estudo epidemiológico da tuberculose no estado de Minas Gerais. Revista Eletrônica Acervo Saúde; 2018; 18: 78.

15. OLIVEIRA AB e MARTINELLI JM. Casos notificados de malária no Estado do Pará, Amazônia Brasileira, de 1998 a 2006. Epidemiologia e Serviços de Saúde, 2009; 18(3): 277-284.

16. PANTOJA GMT e ANDRADE RF. Impactos socioambientais decorrentes dos projetos hidrelétricos na bacia do Rio Araguari: do aumento populacional a disseminação da malária. Planeta Amazônia: Revista Internacional de Direito Ambiental e Políticas Públicas, 2012; (4): 61-74.

17. PARISE EV. Perfil epidemiológico da malária no Estado do Tocantins no período 2003 a 2008. Dissertação (Mestrado Profissional em Ciências da Saúde) - Universidade Federal do Tocantins, Palmas, 2009.

18. PINTO LF. De Tucuruí a Belo Monte: a história avança mesmo? Boletim do Museu Paraense Emílio Goeldi. Ciências Humanas. Belém, 2012; 7(3), 777-782.

19. REY L. Bases de Parasitologia Médica. 3 ed. rev. e atual. - Rio de Janeiro: Guanabara Koogan, 2010.

20. RODRIGUES PT. Origem e rotas de introdução de Plasmodium vivax e Plasmodium falciparum nas Américas. (Tese de Doutorado) - Universidade de São Paulo, São Paulo, 2017.

21. SARMENTO RA, et al. Determinantes socioambientais e saúde: O Brasil rural versus o Brasil urbano. Tempus Actas de Saúde Coletiva, 2015; 9(2): 221 - 223.

22. SUAREZ-MUTIS MC e COURA JR. Mudanças no padrão epidemiológico da malária em área rural do médio Rio Negro, Amazônia brasileira: análise retrospectiva. Cad. Saúde Pública, Rio de Janeiro, 2007; 23(4): 795-804.

23. VICENTE JA. importância da epidemiologia para o enfermeiro. Anais da Semana Acadêmica e Mostra Científica de Enfermagem, 2016; 1.

24. YESHIWONDIM AK, et al. Spatial analysis of malaria incidence at the village level in areas with unstable transmission in Ethiopia. International Journal of Health Geographics, 2009; 8(1): 5 - 10. 